\title{
Golf Training System Using Sonification and Virtual Shadow
}

\author{
Atsuki Ikeda \\ ikeda.a.ad@m.titech.ac.jp \\ Tokyo Institute of Technology \\ Tokyo, Japan
}

\author{
Dong-Hyun Hwang \\ hwang.d.ab@m.titech.ac.jp \\ Tokyo Institute of Technology \\ Tokyo, Japan
}

\section{Homare Kon \\ kon.h.aa@m.titech.ac.jp \\ Tokyo Institute of Technology \\ Tokyo, Japan}

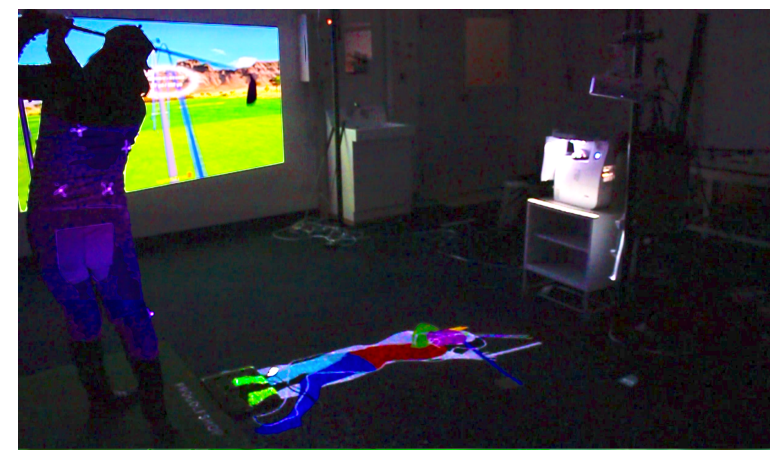

Figure 1: An overview of the system

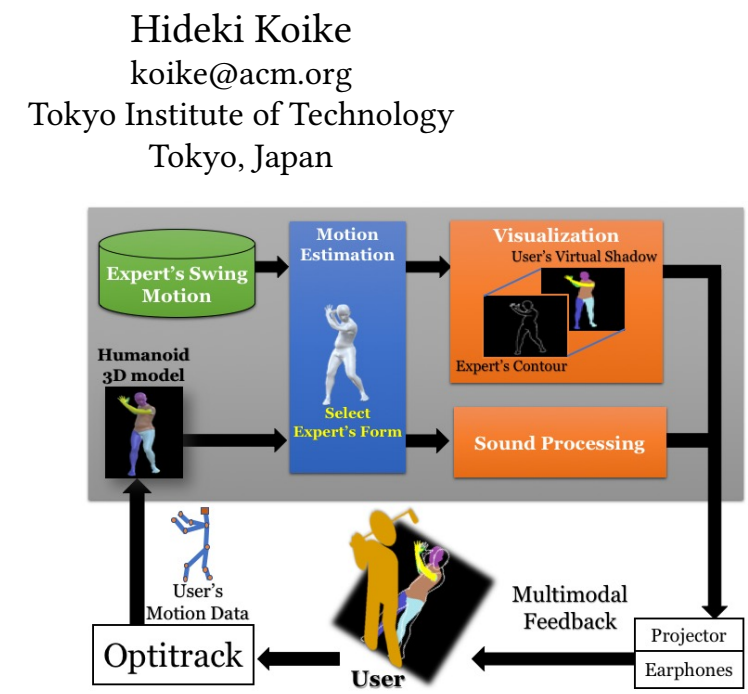

Figure 2: Schematic diagram of the system

\begin{abstract}
This paper proposed a golf training system using real-time audiovisual feedback. The system captures userâĂŹs motion with an optical motion tracking system and projects his/her posture as a virtual shadow on the ground. Unlike other golf training systems, our system enables the user to keep his/her gaze on the ball. The model swing motion of the expert golfer is also overlaid so that the user can understand the difference between his/her motion and expertâĂŹs. Moreover, the system provides audio feedback using sound image localization. It enables the user to understand the position and orientation of the club face, which is often out of sight of the user during his/her swing motion.
\end{abstract}

\section{CCS CONCEPTS}

- Human-centered computing $\rightarrow$ Auditory feedback; Information visualization.

\section{KEYWORDS}

sports training, shadow, golf, sonification, multimodal feedback

Permission to make digital or hard copies of part or all of this work for personal or classroom use is granted without fee provided that copies are not made or distributed for profit or commercial advantage and that copies bear this notice and the full citation on the first page. Copyrights for third-party components of this work must be honored. For all other uses, contact the owner/author(s).

SIGGRAPH '19 Emerging Technologies, July 28 - August 01, 2019, Los Angeles, CA, USA (C) 2019 Copyright held by the owner/author(s)

ACM ISBN 978-1-4503-6308-2/19/07.

https://doi.org/10.1145/3305367.3327993

\section{ACM Reference Format:}

Atsuki Ikeda, Yuka Tanaka, Dong-Hyun Hwang, Homare Kon, and Hideki Koike. 2019. Golf Training System Using Sonification and Virtual Shadow. In Proceedings of SIGGRAPH '19 Emerging Technologies. ACM, New York, NY, USA, 2 pages. https://doi.org/10.1145/3305367.3327993

\section{INTRODUCTION}

In most sports, including golf, skills can be improved by imitating the motion of experts. Many systems have been proposed to teach users the correct swing form with real-time visual feedback. These systems, however, require the users to move their head to see the display, which disturbs the users focusing on the ball. Ikeda's system [Ikeda et al. 2018b] projected the virtual shadow on the ground, which enables the user to obtain feedback with natural head orientation. In addition, the Shadow Drill is often used in golf training.

One of other remaining issues is that user cannot visually recognize the position and orientation of the golf club when the user swings back. Sigrist et al.[Sigrist et al. 2015] reported that audio feedback is more effective than haptic feedback when used in conjunction with visual feedback in trunk-arm rowing.

This paper proposed a golf training system using real-time audiovisual feedback.

\section{PROPOSED SYSTEM}

The proposed system projects the forms of both the user and the expert on the ground in real-time like a shadow as in Figure 3, and 


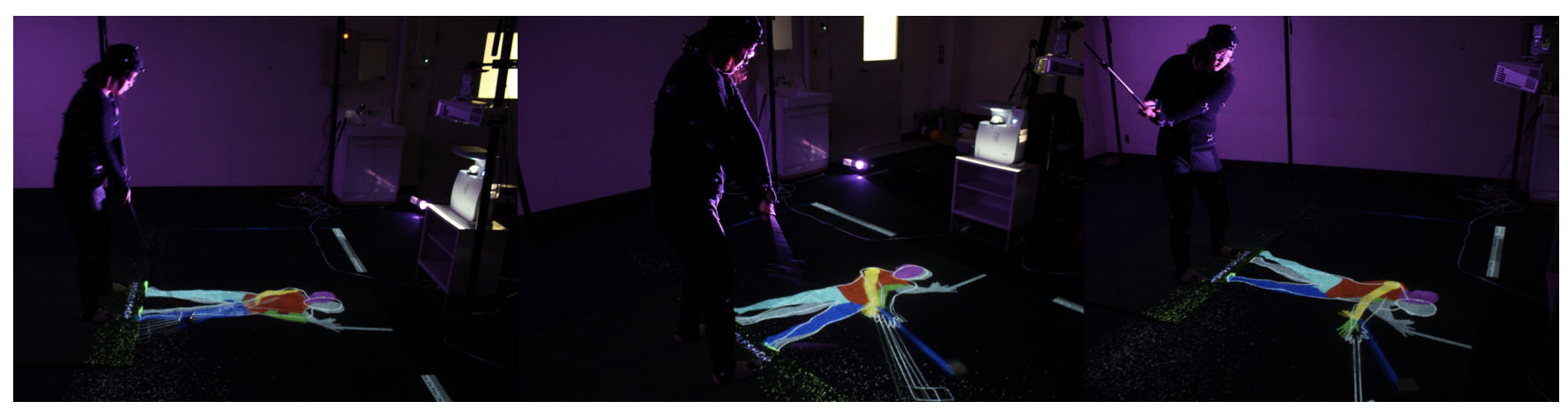

Figure 3: Real-time visual feedback with virtual shadow.

also provides the audio feedback to correct the posture of the club. The shadow visualization enables users to recognize the difference between their form and the expert's. Moreover, the audio feedback makes users understand the differences of the angle and rotation of the club which are difficult to understand only with 2D visual feedback. Our system uses sound image localization which makes users feel as if the sound came from the club face, so that the user can intuitively recognize the position of the club face even if the club is out of sight.

Figure 2 illustrates the system configuration of the system. The system captures the user's form using Optitrack ${ }^{1}$ and projects the virtual shadow using a short-focus projector. The audio feedback is given to the user through the Bluetooth earphone.

\subsection{Visual Feedback}

The virtual shadow of the user is made from the silhouette of SMPL[Loper et al. 2015] model which is a realistic 3D model of the human body. The model is adjusted to the user's form in real-time. In addition, the system projects the outline of the expertâĂŹs form on the shadow. The form of the expert is selected from one motion acquired in advance. DecayedDTW [Ikeda et al. 2018a] is used for this selection.

\subsection{Audio Feedback}

Based on the form of the expert selected above, audio feedback is generated. The audio feedback is provided to the user based on the roll and yaw of the golf club as shown in the Figure 4.

We used white noise and whining noise as audio feedback based on our previous study [Tanaka et al. 2018]. Specifically, based on the sawtooth wave (basic sound), the white noise is added if there is a difference in the roll. The whining noise is added if there is a difference in the yaw. In addition, for sound image localization, the audio feedback is converted to binaural sound through the head-related transfer function [GARDNER 1994].

\section{CONCLUSION}

This paper proposed a golf training system with real-time audiovisual feedback, which does not require the users to shift their gaze direction. The visual feedback is projected on the feet of the user and imitates the shadow. The audio feedback is provided to understand

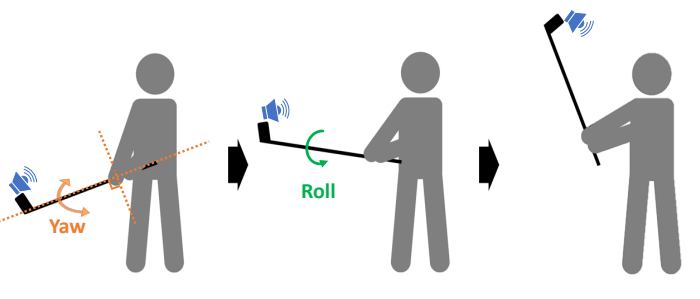

Figure 4: Transition of sound source position by sound image localization and measurement method of two angles (yaw, roll).

the position and orientation of the club face, and compensates information that is lost in 2D visual feedback. In the future, we will conduct a user study of the proposed system and evaluate the effectiveness of this multimodal feedback

\section{ACKNOWLEDGMENTS}

This work was supported by JST CREST Grant Number JPMJCR17A3, Japan.

\section{REFERENCES}

Bill GARDNER. 1994. HRTF Measurements of a KEMAR Dummy-Head Microphone. http://sound.media.mit.edu/KEMAR.html (1994). https://ci.nii.ac.jp/naid/ 10022493601/

Atsuki Ikeda, Dong Hyun Hwang, and Hideki Koike. 2018a. AR based Self-sports Learning System using Decayed Dynamic TimeWarping Algorithm. In ICAT-EGVE 2018 - International Conference on Artificial Reality and Telexistence and Eurographics Symposium on Virtual Environments, Gerd Bruder, Shunsuke Yoshimoto, and Sue Cobb (Eds.). The Eurographics Association. https://doi.org/10.2312/egve.20181330

Atsuki Ikeda, Dong-Hyun Hwang, and Hideki Koike. 2018b. Real-time Visual Feedback for Golf Training Using Virtual Shadow. In Proceedings of the 2018 ACM International Conference on Interactive Surfaces and Spaces (ISS '18). ACM, New York, NY, USA, 445-448. https://doi.org/10.1145/3279778.3279927

Matthew Loper, Naureen Mahmood, Javier Romero, Gerard Pons-Moll, and Michael J. Black. 2015. SMPL: A Skinned Multi-person Linear Model. ACM Trans. Graph. 34, 6, Article 248 (Oct. 2015), 16 pages. https://doi.org/10.1145/2816795.2818013

Roland Sigrist, Georg Rauter, Laura Marchal-Crespo, Robert Riener, and Peter Wolf. 2015. Sonification and haptic feedback in addition to visual feedback enhances complex motor task learning. Experimental Brain Research 233, 3 (01 Mar 2015), 909-925. https://doi.org/10.1007/s00221-014-4167-7

Yuka Tanaka, Homare Kon, and Hideki Koike. 2018. A Real-time Golf-swing Training System Using Sonification and Sound Image Localization. In Proceedings of the 24th ACM Symposium on Virtual Reality Software and Technology (VRST '18). ACM, New York, NY, USA, Article 66, 2 pages. https://doi.org/10.1145/3281505.3281604 